\title{
Characterization of $\mathbf{N}$-Acetylcysteine Conjugate in Yellow Urine by Oral Administration of 1,4-Dichloro-2-Nitrobenzene in Rats
}

\author{
Makoto Ohnishi,* Kazunori Yamazaki, Seigo Yamamoto, and Taijiro Matsushima \\ Japan Bioassay Research Center, Japan Industrial Safety and Health Association, 2445 Hirasawa, Hadano-shi, Kanagawa 257-0015, \\ Japan
}

(Received March 8, 2004; Accepted March 23, 2004)

The color of urine from rats fed 1,4-dichloro-2nitrobenzene (DCNB) is a clear yellow than that of urine from control rats. In order to obtain information on the relationship between the yellow color of the urine and its constituents, analyses of the components in the urine from rats fed DCNB were performed by UV, nuclear overhauser effect (NOE) $\operatorname{method~of~}{ }^{1} \mathbf{H}$ NMR and liquid chromatography (LC)-MS/MS. The urine from the rats fed DCNB had absorbance at a wavelength of $383 \mathrm{~nm}$. Characteristic fragment ions of the $N$-acetylcysteine conjugates, at $m / z 187$, were clearly observed in the fragmentation of the precursor ions at $m / z 317$ ([M-H $\left.]^{-}\right)$by LC-MS/MS. And position of the structure was found to be $\mathrm{N}$-acetyl-S-(4chloro-3-nitrophenyl)- $L$-cysteine by NOE method. Consequently, in view of the conformity of the major constituent ( $N$-acetylcysteine conjugates), when comparing yellow urine and the metabolite, it is thought that the metabolite formation is attributable to the longer wavelength absorbance of the $\mathrm{N}$-acetyl-S-(4chloro-3-nitrophenyl)- $L$-cysteine, as compared to the control urine.

Key words — 1,4-dichloro-2-nitrobenzene, metabolites, $N$-acetylcysteine conjugate, rat, yellow urine, liquid chromatography-MS/MS

\section{INTRODUCTION}

1,4-Dichloro-2-nitrobenzene (DCNB), a halogenonitrobenzene, is commonly used in industrial intermediates as an organic dye. A few studies on the metabolism of DCNB have been reported. ${ }^{1)}$ DCNB is metabolized to glutathione conjugates,

\footnotetext{
*To whom correspondence should be addressed: Japan Bioassay Research Center, Japan Industrial Safety and Health Association, 2445 Hirasawa, Hadano-shi, Kanagawa 257-0015, Japan. Tel.: +81-463-82-3911; Fax: +81-463-82-3860; E-mail: monishi@jisha.or.jp
}

resulting in the production of $N$-acetylcysteine conjugates as the major metabolite. It is well known that mercapturic acid is a conjugate of $\mathrm{N}$-acetylcysteine and is the end product of an important process in the body for the probable detoxication of a variety of potentially harmful electrophilic compounds with the endogenous nucleophile glutathione. ${ }^{2-4)}$ Bray et al. have carried out extensive studies of the metabolism of halogenonitrobenzenes in rabbits; the results show that these compounds are biotransformed to mercapturic acids. ${ }^{5)}$ Recently, the Japan Bioassay Research Center investigated the carcinogenicities on several kinds of halogenonitrobenzenes. DCNB was one of these chemicals, and the color of urine from the rats fed DCNB was a clear yellow than that of the urine from the control rats.

Our research aim to clarify a relationship between yellow color of the urine and exposure of DCNB and give a information of bio-marker to monitor worker's exposure. The structural characteristics of the urinary metabolite in rats remain to be clearly elucidated, including the two chlorine substitutes for it. Analyses of the components of $N$ acetylcysteine conjugate in urine from the rats fed a diet containing DCNB were performed by, UV, ${ }^{1} \mathrm{H}-$ NMR and liquid chromatography (LC)-MS/MS.

\section{MATERIALS AND METHODS}

Chemicals — DCNB, HPLC-grade acetonitrile and distilled water were obtained from Wako Pure Chemical Ind. (Osaka, Japan).

Animals and Their Maintenance _- Male F344/DuCrj rats were purchased from Charles River Japan, Inc. (Atsugi, Japan). The animals were housed in polycarbonate metabolic cages. Room temperature and the relative humidity were controlled at 23 $\pm 1^{\circ} \mathrm{C}$ and $58 \pm 8 \%$, respectively. 
Diet Preparation — A diet containing DCNB was prepared by mixing DCNB into CRF-1 powdered diet (Oriental Yeast Co., Ltd., Chiba, Japan) in a spiral mixer for $20 \mathrm{~min}$. A group of 3 male rats were fed the diet containing 1.0\% DCNB for 2 days. Sample Collection of Urine — Urine samples were collected from 3 rats fed the DCNB-containing diet in polycarbonate metabolic cages for $24 \mathrm{hr}$, and then were pooled as one sample.

Sample Preparation for UV Analysis of Urine - The urine from the rats fed DCNB and the urine from the control rats were separately dissolved in distilled water. The UV spectra of these samples were obtained by measurement with a UV spectrometer.

Sample Preparation for ${ }^{1} \mathrm{H}-\mathrm{NMR}$ and LC-MS/MS Analyses of Urine — The $\mathrm{pH}$ of eight milliliters of urine was adjusted to $1.2-1.3$ by adding $2 \mathrm{ml}$ of $10 \%$ trifluoroacetic acid. The yellow component was extracted using Oasis HLB columns from Waters Corp. (Milford, U.S.A.), which were pretreated with $3 \mathrm{ml}$ acetonitrile and then $3 \mathrm{ml}$ water. The water in the column was removed by centrifugation (400 $\mathrm{g}$ for $10 \mathrm{~min}$ ), and the yellow component was subsequently eluted by $3 \mathrm{ml}$ acetonitrile. The elute was evaporated under vacuum at $40^{\circ} \mathrm{C}$ to dryness the residue. The residue dissolved in the mobile phase of LC-MS/MS was used for LC-MS/MS analyses of the DCNB conjugates. Residue dissolved in deuterated methanol was used for ${ }^{1} \mathrm{H}-\mathrm{NMR}$ analyses of the DCNB conjugates.

UV and ${ }^{1} \mathbf{H}-N M R$ Analysis — UV analysis was performed using a UV-2400PC, Shimadzu (Kyoto, Japan). ${ }^{1} \mathrm{H}-\mathrm{NMR}$ analysis was performed using a DPX 300, Bruker (Karlsruhe, FRG) by the nuclear overhauser effect (NOE) method ${ }^{6,7)}$ for the DCNB conjugates

LC-MS/MS Analysis — A prepared sample of urine was analyzed by LC-MS/MS. LC-MS/MS analyses were performed using an HP 1090 from Hewlett-Packard (Waldbronn, FRG) and a TSQ 7000 from Thermo Quest (San Jose, U.S.A.). HPLC was carried out using flow injection at a flow rate of $0.2 \mathrm{ml} / \mathrm{min}$. The mobile phase was methanol- $0.02 \%$ acetic acid $(3: 2, \mathrm{v} / \mathrm{v})$. The mass spectrometer was operated in the negative ion mode with a potential of $4.5 \mathrm{kV}$ applied to the electrospray needle. Dried nitrogen gas was used as the sheath gas (70 psi) and auxiliary gas (5 units) to assist with nebulization. The capillary temperature was held at $200^{\circ} \mathrm{C}$.

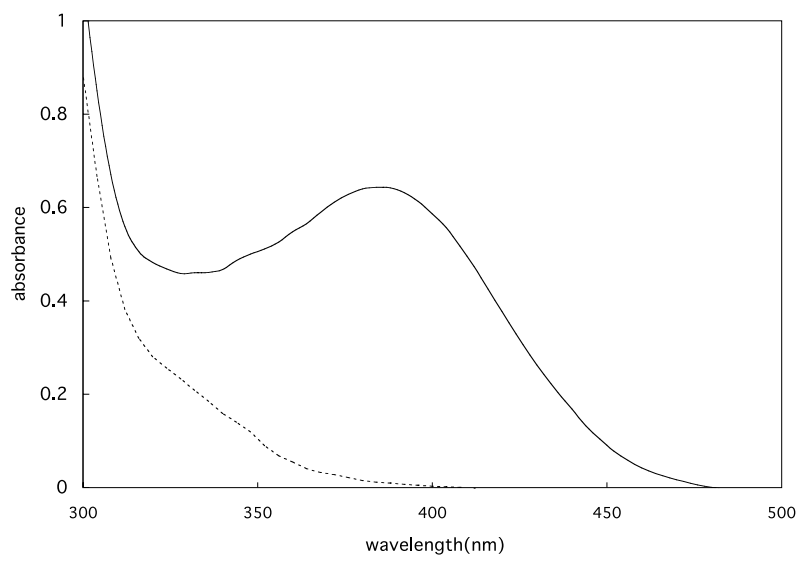

Fig. 1. UV Spectrum of Urine from Rats Fed the Powdered Diet Containing 1,4-Dichloro-2-Nitrobenzene (Solid Line) and Urine from Control Rats (Dotted Line)

\section{RESULTS AND DISCUSSION}

It is obvious that the color of urine from the rats fed DCNB is a clear yellow than that of the urine from the control rats. Figure 1 shows the UV spectra obtained for the yellow urine from the rats fed DCNB, and urine from the control rats. The UV spectrum of the yellow urine showed a broad peak at a wavelength of $383 \mathrm{~nm}$. On the other hand, a peak for the urine from the control rats was not observed at this wavelength. This result implies that the major components of yellow urine is the metabolite of DCNB. Booth and Boyland studied glutathion conjugate formation of 1,2-dichloro-4-nitrobenzene, isomeric form of DCNB, by glutathione $S$-aryltransferase and showed absorption spectrum changes to a longer wavelength. ${ }^{8)}$ It should be noted that the shift of the longer wavelength of the glutathione conjugate corresponds to the wavelength shift of the urine metabolite of DCNB.

Figure 2 shows the NOE spectrum of the metabolite and the chemical structure of proton position. The two NOE peaks observed at the crossing position between $\mathrm{H}-\mathrm{b}, \mathrm{H}-\mathrm{c}$ and $\mathrm{H}-\mathrm{d}$ indicated that the proton $\mathrm{H}-\mathrm{d}$ is located the near position of proton $\mathrm{H}-\mathrm{b}$ and $\mathrm{H}-\mathrm{c}$. This result suggested that the s-conjugate clorine is located at not 1-position but 4-position of benzene ring. The position of the metabolite shows the presence of three protons, $\mathrm{H}-\mathrm{a}$ (8.14 ppm; doublet peaks), H-b (7.67 ppm; singlet peak) and Hc (7.66 ppm; doublet peaks), respectively, suggesting hydrogen of the aromatic ring. The characteristics of the proton $\mathrm{H}$-d of $\mathrm{N}$-acetylcysteine suggest two double doublet peaks at $3.27-3.35 \mathrm{ppm}$ and at 


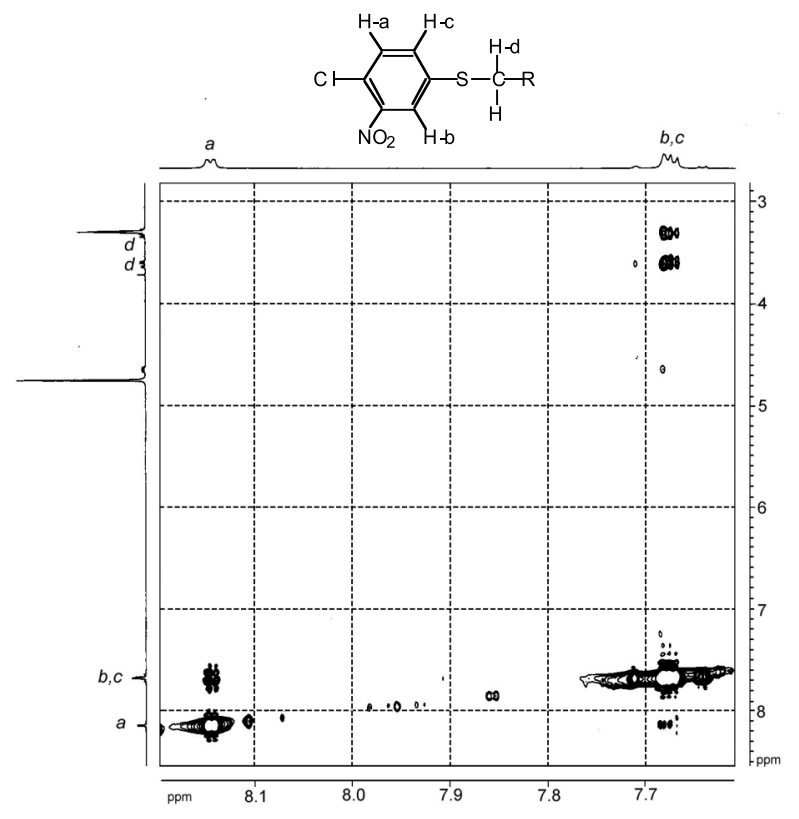

Fig. 2. NOE Spectrum of a Urinary Metabolite from Rats Fed the Powdered Diet Containing 1,4-Dichloro-2Nitrobenzene

3.58-3.65 ppm. The two NOE peaks were accepted by the crossing position between protons $\mathrm{H}-\mathrm{b}, \mathrm{H}-\mathrm{c}$ and proton $\mathrm{H}-\mathrm{d}$ and this suggesting that the proton $\mathrm{H}-\mathrm{d}$ is near the protons $\mathrm{H}-\mathrm{b}$ and $\mathrm{H}-\mathrm{c}$.

Figure 3 shows the MS spectrum of the metabolite. The results of analysis for the MS spectrum suggest that the metabolism of DCNB resulting in the formation of $N$-acetylcysteine conjugates follows the process: DCNB $\rightarrow$ glutathione conjugates $\rightarrow N$ acetylcysteine conjugates. Characteristic fragment ion of the $\mathrm{N}$-acetylcysteine conjugate was clearly observed in the fragmentation of the precursor ion at $m / z 317\left([\mathrm{M}-\mathrm{H}]^{-}\right)$and products ions at $\mathrm{m} / z 187$ of $N$-acetyl-S-(4-chloro-3-nitrophenyl)- $L$-cysteine and it's isotope ion of chlorine at $m / z 189$. The product ion at $m / z 187\left([\mathrm{M}-131]^{-}\right)$was in agreement with the calculation value of $\mathrm{N}$-acetylcysteine moiety from $N$-acetyl-S-(4-chloronitrophenyl)- $L$-cysteine. The chemical structure of $\mathrm{N}$-acetyl-S-(4-chloro-3nitrophenyl)- $L$-cysteine is shown in Fig. 3. Therefore, the structure of DCNB conjugates in the urine from rats fed the diet containing DCNB was found to be $N$-acetyl-S-(4-chloro-3-nitrophenyl)- $L$-cysteine by LC-MS/MS method.

Consequently, in view of the conformity of the major constituent ( $N$-acetylcysteine conjugates), when comparing yellow urine and the metabolite, it is thought that the formation of metabolite is attributable to the longer wavelength of the $N$-acetyl- $S$ -

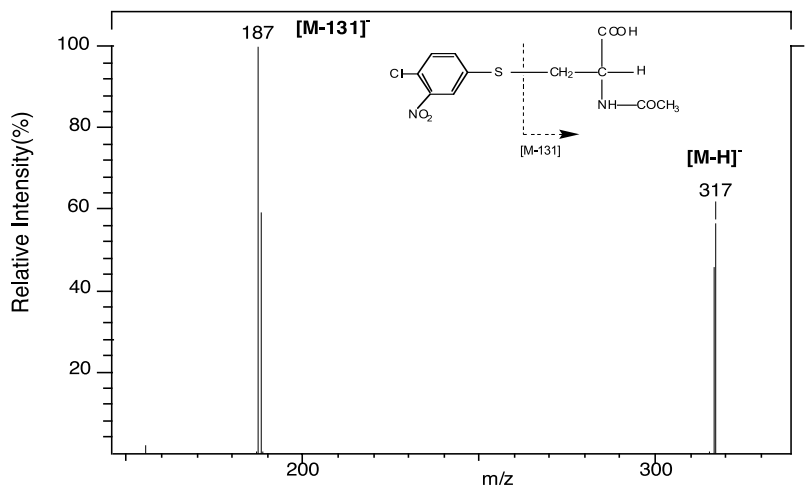

Fig. 3. LC-MS/MS Spectrum of a Urinary Metabolite from Rats Fed the Powdered Diet Containing 1,4-Dichloro-2Nitrobenzene

(4-chloro-3-nitrophenyl)- $L$-cysteine, as compared to the control urine. The analysis of $\mathrm{N}$-acetyl-S-(4chloro-3-nitrophenyl)- $L$-cysteine having a broad absorption peak at $383 \mathrm{~nm}$ could serve as a biomarker for exposure of workers to DCNB.

Acknowledgements We thank Dr. Misako Imachi (Bruker Biospin K.K., Ibaraki, Japan) for technical assistance with ${ }^{1} \mathrm{H}-\mathrm{NMR}$.

\section{REFERENCES}

1) Bray, H. G., James, S. P. and Thorpe, W. V. (1957) The metabolism of 2:4-, 2:5- and 3:4-Dichloronitrobenzene in the rabbit. Biochem. J., 65, 483-490.

2) Barnes, M. M., James, S. P. and Wood, P. B. (1959) The formation of mercapturic acids. 1. Formation of mercapturic acid and the levels of glutathione in tissues. Biochem. J., 71, 680-690.

3) Bray, H. G., Franklin, T. J. and James, S. P. (1959) The formation of mercapturic acids. 2. The possible role of glutathionase. Biochem. J., 71, 690-696.

4) Boyland, E. and Chasseaud, L. F. (1969) The role of glutathione and glutathione s-transferases in mercapturic acid biosynthesis. Adv. Enzymol., 32, 173219.

5) Bray, H. G., James, S. P. and Thorpe, W. V. (1957) The metabolism of 2:3-, 2:6- and 3:5-dichloronitrobenzene and the formation of a mercapturic acid from 2:3:4:5-tetrachloronitrobenzene in the rabbit. Biochem. J., 67, 607-616.

6) Kaburagi, T., Yoshitsugu, H., Uekusa, H., Ishibashi, M. and Nanbo, T. (2000) Identification and clearance involved in the formation of glucuronides of RT3003, a new peripheral blood flow enhancer, and its metabolite in rats. Biol. Pharm. Bull., 23, 128-131. 
7) Breyer-Pfaff, U., Prox, A., Wachsmuth, H. and Yao, P. (1987) Phenolic metabolites of amitriptyline and nortriptyline in rat bile. Drug Metab. Dispos., 12, 882-889.
8) Booth, J., Boyland, E. and Sims, P. (1961) An enzyme from rat liver catalysing conjugations with glutathione. Biochem. J., 79, 516-524. 